Vol. 1, No. 2, December 2020

DOI: https:/ / doi.org/10.18196/ijiep.v1i2.10011

\title{
The Impact of Social Environment on The Sexual Self- Regulation of University Students in Uganda
}

\author{
${ }^{1}$ Musa Muwaga, ${ }^{2}$ Fuad Nashori ${ }^{*},{ }^{2}$ Achmad Sholeh \\ ${ }^{1}$ Department of Psychology, School of Arts and Social Sciences, Kampala University, Uganda \\ ${ }^{2}$ Department of Psychology, Faculty of Psychology and Socio-Cultural Sciences, Universitas \\ Islam Indonesia, Indonesia
}

*Corresponding email: fuadnashori@uii.ac.id

\section{ARTICLE INFO}

Article history

Received, 12/10/2020

Revised, 13/11/2020

Accepted, 08/12/2020

Keywords

Attitudes

Sexual Self-Regulation

Social Environment

Religious Values

University Students

\begin{abstract}
Most societies consider sexual self-regulation to represent a general temptation control mechanism, postponing gratification. This study aims to determine the social environment's impact on university students' sexual self-regulation in Uganda. The data were obtained using convenience technique sampling of undergraduate students at university " $\mathrm{X}$ " in Uganda. Students voluntarily completed the questionnaire in a regular classroom setting. The questionnaire elicited information regarding sexual regulative capacities like seeking accurate information from social environments like family, peer, and university environments. The data were analyzed using both univariate analyses (chi-square and analysis of variance and logistic regression. The results indicated that the social environment had a positive and significant relationship with sexual self-regulation and university students with a value of $1 \mathrm{t} 1 \geq 1.96$, and factor loading $\geq 0.50$ was significant. These results should be considered by those working with university students in human sexuality and human development.
\end{abstract}

This is an open access article under the CC-BY-SA license.

Citation:

Muwaga, M., Nashori, F. \& Sholeh, A. (2020). The Impact of Social Environment on The Sexual Self-Regulation of University Students in Uganda. International Journal of Islamic Educational Psychology, 1(2), 75-88. DOI: https:/ / doi.org/10.18196/ijiep.v1i2.10011 
Muwaga, Nashori \& Sholeh | The Impact of Social Environment on The Sexual Self-Regulation of University Students in Uganda

\section{INTRODUCTION}

According to the United Nations International Children's Emergency Fund (UNICEF), adolescents can be categorized by three stages: 10-13 years old as early adolescents', 14-16 years old as middle adolescents', and 17-19 years old as late adolescents.

Based on the developmental perspective, adolescence is a very challenging time with various conflicts. Physically, adolescents' bodies are getting mature, then, cognitively, they tend to learn of a different future, and emotionally, shifting relationships with family and peers plays a crucial role in defining their experiences (Lauren \& Sharlene, 2012).

Adolescence is one of life's most fascinating and complex transitions, a time of accelerated growth and alters second solely to infancy; a time of broadening horizons, self-discovery, and emerging independence, a time of transformation from childhood to adulthood, helping to begin is associated with profound biological, physical, behavioral, and social transformations that roughly correspond with the move to university or higher education. In these confusing years, young people experience a great deal of development, excitement, doubt and confusion (Blanchard, Zucker, Siegelman, Dickey, Klassen, 1998). Relationships between family and peers provide a new significance. Others have little more than a blurred picture of the future as they embark on a protracted quest for paths to promising adulthood.

In cultures worldwide, adolescents' onset is closely synchronized with the hormonal changes in puberty (Fried, 2015). Today, in the most technologically sophisticated nations, puberty, on average, starts two years earlier than it did a century ago, and the transition to maturity will take ten years or longer. Early adolescence, which includes the sexual and psychological awakening of puberty and new social struggles, spans from about 10 to 14 years of age (Frost \& Meyer, 2009). Middle puberty, a period of increased autonomy and creativity, is between fifteen and seventeen. Late puberty, occurring among those who defer their entrance into adult positions due to educational or social reasons, can vary from 18 to 20 years of age. A time of opportunity and risk for most young people, the teenage transition provides a great opportunity and excitement, if also hardship and disappointment.

Some students have noted that, contrary to common wisdom, adolescents as a group are not necessarily troublesome, in comparison, and victims of "hormone hype" for many, experience is surprisingly smooth, mainly where family relationships are based on an appreciation of adolescent developmental needs. Overall, teens' supportive relationships with their families are much less stormy than commonly believed (Iskandar, Setiono, Agustiani, \& Muwaga, 2013).

In vital adolescent years, most parents have a significant influence on their offspring, helping shape their sense of self and influence their possible life decisions. Peers also have a positive effect on an adolescent, contributing to their 
Muwaga, Nashori \& Sholeh | The Impact of Social Environment on The Sexual Self-Regulation of University Students in Uganda

self-esteem (Mujiyati \& Adiputra, 2018), sense of identity (Ragelienè, 2016), and achievement (Hayes et al., 2015; Mikami et al., 2017). Peer support is often extremely relevant for those whose parents are emotionally distant, strongly negative, or casually neglectful. Despite reassuring results, teenagers face demands and desires and risks and dangers more frequent and more severe than they were a century earlier. Millions are growing up under conditions that do not satisfy their long-term requirement for ideal development. They give way to peer forces to use drugs and alcohol and cigarettes, to have intercourse and participate in antisocial behaviors (Iskandar et al., 2013).

According to Ridge and Feeney (1998), amid serious efforts and investment in providing sexual knowledge, there is only minimal impact among young people. Awareness about sexual activity by most adolescents' 'little' myths and misconception of sexuality, pregnancy, and sexual health are widespread among adolescents. As the conversation about sex or sexual education is something "taboo", especially in several countries such as Asia. Some skeptics argue that it encourages promiscuity among young people and believes that this problem should be avoided to "wake sleeping bears" (Leung, Shek, Leung, \& Shek, 2019). Adolescents face legal, social and cultural challenges to accessing factual and useful knowledge about sexual behavior. The knowledge they get from the textbooks is not enough to address the complicated problems of daily life. Social media, such as daily newspapers, television, the internet, and radio, are not credible sources of knowledge and have become critical secondary sources when teenagers are anxious to obtain correct and reliable responses about their sexual behavior.

Sexual self-regulation is how adolescents regulate their sexuality (Iskandar et al., 2013). To date, research that supports adolescents' intrinsic and extrinsic sexual self-regulative capacities, particularly in the domain of psychology, is nonexistent. Having sexual self-regulative ability during adolescence is an invaluable tool that is only rarely given due focus in conventional psychological research (Iskandar et al., 2013).

Based on the findings available from different researchers, they are always quick to conclude that adolescents' sexual behaviors increase due to changes in a social environment consisting of family, peers and learning institutions (Iskandar et al., 2013; Ridge \& Feeney, 1998). Social environments are dynamic and change over time as the result of both internal and external forces. There are relationships of dependency among different local areas' social environments because these areas are connected through more extensive regional, national, and international social and economic processes and power relations (Barnett \& Casper, 2001). The changes in the social environment have also been related to changes in the economy, where the higher number of parents working outside the home has more than doubled since the 1970s. The interaction of changes in social environment and employment has increased the amount of time that adolescents spend unsupervised by adults, yet others as they advance in their educational 
Muwaga, Nashori \& Sholeh | The Impact of Social Environment on The Sexual Self-Regulation of University Students in Uganda

career; they get separated from their immediate yet significant persons in their lives, therefore increasing the time that they spend alone or with peers. The problems with unsupervised time of adolescents are at least two. First, it provides ample opportunities for adolescents to engage in behaviors that adults might disapprove of or prohibit if they were present. These include behaviors that must be appealing to adolescents', such as alcohol use and sexual intercourse linked to adulthood, maturity, and independence, yet is also health-compromising (Fried, 2015; Noviva \& Wahyono, 2020). The developmental need of adolescents for independence and their desire to attain adult status and maturity provide an incentive for them to engage in what they see as adult behaviors. More significant unsupervised time may prematurely grant both independence and emotional emancipation from the family. In the other hand, a prolonged positive atmosphere for adolescents, with incremental moves towards autonomy, tends to improve the health behavior of adolescents (Fried, 2015).

However, the researcher has not come across data or research findings that show changes in the social-environment. Simple surveys use single value approaches that do not give comprehensive or detailed explanations about the social environment's functioning with adolescents' sexual behaviors. The researcher appreciates available research findings concerning adolescents' development and their sexual behaviors. However, there's a need for more studies to explore how the social environment influences adolescents' sexuality. There are all reasons to believe that the increase in sexuality among adolescents is due to failures of sexual self-regulation among adolescents and not only failures or changes in the social environment alone.

The study conducted by Iskandar et al. (2013) showed the relationship between the social environment and adolescents' sexual self-regulation, affecting sexual self-regulation. Adolescents' lacked the skills to structure their environment, seeking assistance from significant others, for example, parents and university environment. They also revealed that adolescents' lacked the efficacy to stick to their set goals and came to conform to peers' pressure and weak self-efficacy to control their emotions concerning sexual desires.

In this research, the social environment will investigate from the point of view of Bandura, which argued that the family, peers and learning institutions have a significant impact on adolescent behavior and that the family is the most important venue for social interaction and participation, "with parents playing a vital role in the schema of adolescent behavior, even though the impact of the family gets weaker in middle adolescence because the center of a youth's activities step by step moves from home to high education and Peer networks, the trend towards decreasing communication effects between parents and their children continues as adolescents enter later adolescence when new forms of commitment to conventional activities replace attachment and communication to parents and become a significant source of the bond to the traditional society for young adults. Bandura proposes that individuals follow right and wrong 
Muwaga, Nashori \& Sholeh | The Impact of Social Environment on The Sexual Self-Regulation of University Students in Uganda

principles, which serve as guides and disincentives for self-creation behavior. In this regulatory process, people monitor their conduct and the conditions under which it takes place, judge it about moral standards and regulate their behavior by their consequences. Individuals do what gives them happiness and a sense of self-worth. Individuals renounce actions that contradict their values because such behavior can lead to self-condemnation. Self-sections shall be performed in compliance with national guidelines (Iskandar et al., 2013). This study examines the impact of the social environment on university students' sexual selfregulation in Uganda.

\section{METHODS}

Participants

The participants in this study were 408 undergraduate students at Kampala University. The research participation was voluntary, and all participants remained anonymous. All students attending classes on days data were gathered and allowed to participate in the analysis. Few students refused to participate; the approximate figure was more than $90 \%$.

\section{Data Collection}

Data was obtained using convenience technique sampling. The data collection method was carried out by the scale of sexual self-regulation, the social environment, and many demographic factors. For this research's objectives, students were asked about their participation (yes or no) in the following sexual self-regulation behavior: 1) sexual intercourse, necking, petting, 2) sexual intercourse in the last month. These items are commonly used in studies regarding sexual behaviors; relatively few studies have examined sexual selfregulation. Social environment factors such as families, peers and the university environment were also used in the questionnaire. Adequate reliability of items (Cronbach's alpha $=.63$ ) was recorded in previous studies, where items were used as a single six-point scale representing participants' understanding of the social environment's view of sexual self-regulation. Since the researcher was studying sexual self-regulation before marriage, the participant's voluntary questionnaire was conducted within the regular class schedule, and the Institutional Review Board (IRB) 's approval was granted before the study.

\section{Data Analysis}

Data were analyzed using both univariate analyses (chi-square and analysis of variance and logistic regression to investigate the impact of social environment on sexual self-regulation of university students in Uganda.

\section{Results}

This study is intended to test the social environment's impact on university students' sexual self-regulation in Uganda empirically. The result can be seen in the table below. 
Muwaga, Nashori \& Sholeh | The Impact of Social Environment on The Sexual Self-Regulation of University Students in Uganda

Table 4.1 Goodness of fit for Social Environment Model

\begin{tabular}{ccc}
\hline The Goodness of Fit Index (GFI) & Predictive fit index & Estimates \\
\hline Chi-Square (df) & Smaller value & $491.01(443) 1.1087$ \\
P (close fit) & $P \geq 0.05$ & 0.00 \\
RMSEA & RMSEA $\leq 0,08$ & 0.01 \\
RMR & RMR $\leq 0,05$ & 0.04 \\
GFI & GFI $\geq 0,90$ & 0.91 \\
CFI & CFI $\geq 0,90$ & 0.93 \\
\hline
\end{tabular}

When the value of $1 \mathrm{t} 1 \geq 1.96$, then the causal relationship is significant, and from the calculations, the value of $\mathrm{t} l>1.96$ so that the measurement models significant, Factor loading $\geq 0.50$, is said to be very significant, from the calculations, factor loading of aspects were above $\geq 0.50$, with construct reliability of .774 .Therefore, the hypothesis that, social environment influence adolescents sexual behaviors through religious values, sexual self-regulation and attitudes, results indicates that; $x^{2}=1.1087, \mathrm{df}=443, \mathrm{p}-$ Value $=0.00 ; \mathrm{RMSEA}=0.01 ; \mathrm{RMR}=0.04 ; \mathrm{GFI}=0.91$; AGFI $=0.93$.

The result of standardized direct coefficient for an integrated social environment model, the correlation between social environment and religious values emerged as $\left(0.58^{2}\right)$ or $33.64 \%$ of the variance of adolescents religious values are accounted for by social environment which emerged to be statistically significant at $(P<$ $0.05)$, while the remaining $66.36 \%$ of the variance is accounted for by factors outside this study, the correlation between social environment and sexual selfregulation is $\left(0.22^{2}\right)$ or $04.84 \%$ of the variance is accounted for by social environment found not to be statistically significant at $(P>0.05)$, while the remaining $95.16 \%$ of the variance is accounted for by unique factors, correlation of social environment and attitudes is $\left(0.89^{2}\right)$ or $79.21 \%$ of the variance is accounted for by social environment revealed to be statistically significant at $(P<$ 0.05), while 20.79 of the variance is accounted for by other unique factors, these correlations suggests that the variables are somewhat related, as would be expected given the fact that it was hypothesized that these aspects influence adolescents sexual behaviors. However, these correlations are not so high. This result suggests that they are all measuring the same construct; the social environment significantly influences religious values and attitudes but not sexual self-regulation.

Furthermore, the hypothesis test result indicates that the family environment influences adolescents' sexual behaviors through religious values, sexual selfregulation and attitudes. Testing the family environment reveals the goodness of fit measurement with the available data. Therefore, the hypothesis that family environment influence adolescents' sexual behaviors through religious values, sexual self-regulation and attitudes, results indicates that $x^{2}=1,690, \mathrm{df}=300, \mathrm{p}$ value $=0.00 ; \mathrm{RMSEA}=, 049 ; \mathrm{RMR}=.461 ; \mathrm{GFI}=.925 ; \mathrm{CFI}=.940$. 
Muwaga, Nashori \& Sholeh | The Impact of Social Environment on The Sexual Self-Regulation of University Students in Uganda

Table 4.2Goodness of fit for Family Environment Model

\begin{tabular}{ccc}
\hline The Goodness of Fit Index (GFI) & Predictive fit index & Estimates \\
\hline Chi-Square (df) & Smaller value & $507,097(300) 1,690$ \\
P (close fit) & P $\geq 0.05$ &, 000 \\
RMSEA & RMSEA $\leq 0,08$ &, 049 \\
RMR & RMR $\leq 0,05$ &, 461 \\
GFI & GFI $\geq 0,90$ &, 925 \\
CFI & CFI $\geq 0,90$ &, 940 \\
\hline
\end{tabular}

The result of standardized direct coefficient for family as a social environment that influence adolescents sexual behaviors through religious values, sexual selfregulation and attitudes; the coefficient between family environment and religious values is $\left(0.41^{2}\right)$ or $16.81 \%$ of the variance of adolescents religious values are accounted for by family environment and was revealed to be statistically significant at $(P<0.05)$, while the remaining $83.19 \%$ of the variance is accounted for by factors outside this study, the coefficient between family environment and sexual self-regulation is $\left(-0.05^{2}\right)$ or $-2.5 \%$ of the variance is accounted for by family environment not statistically significant at $(P>0.05)$, while the remaining $102.5 \%$ of the variance is accounted for by unique factors, correlation of family environment and attitudes is $\left(0.53^{2}\right)$ or $28.09 \%$ of the variance is accounted for by family environment statistically significant at $(P<0.05)$, while $71.91 \%$ of the variance is accounted for by other unique factors but not family environment.

\section{RESULT AND DISCUSSION}

The results shown in the following paragraphs give a variety of examples of the complex interactions between adolescence and the social environment and how these relationships may have different behavioral roots, serving multiple roles during adolescent development.

The need to transgress societal norms and experience new sensations or new roles is generally more substantial among teens, especially in a family environment. In general, teenagers are less satisfied with communication from their families' implicit information concerning sexual behaviors. They struggle to grasp its utility and feel less confident about their behavior. In reality, they have unpredictable chances of having intercourse because of their present troubled situation of lacking accurate and reliable information from their role models. Based on the fact that expectations characterize teenagers aged 19-21 at University " $X$ " to achieve an educational career, their engagement in sexual behavior of intercourse but not necking and petting are deemed irreproachable.

This situation of low religious values of adolescents aged 19-21 appears to be more multifaceted. While they are somewhat unsatisfied with a family 
Muwaga, Nashori \& Sholeh | The Impact of Social Environment on The Sexual Self-Regulation of University Students in Uganda

environment that provides them with implicit sexual information, results emerged with a significant relationship between adolescents' sexual regulation and sexual behaviors. However, how adolescents develop these capabilities without being externally supported remains a question of debate. This condition is not a remote observation because research findings on how self-regulation develops among adolescents are inconclusive. Some contend that it is externally stimulated, while others believe that it is internally developed and possible that late adolescents are given their formal cognitive development stage and experience outside their social surroundings. They might have got more information about sexual self-regulation, but they put adolescents at a flash to moderately regulate their sexual behaviors because they were obtained outside reliable sources.

Sexual behaviors demonstrate to adolescents and their peers have exceeded their child status and will be embraced by a group of sexually active peers, thereby earning more acceptance and popularity (Rosario, Schrimshaw, Hunter, \& Braun, 2006). Adolescents who have had sexual behaviors spend more time with and receive more generous support from their friends, confirmed in the present study where adolescents are engaged in petting behaviors, having a commitment and spending time together.

Sexual behavior is more deeply felt where teenagers have insufficient social capital, as proved by findings that social settings do not work to their desires and engage with teenagers regarding sexual self-regulation: self-monitoring, selfefficacy, and self-evaluation. For example, adolescents adopted promiscuous sexual behavior patterns of necking and petting because such behaviors are counted less on their social environment, family, university and peers. These adolescents' perspectives provide a significant resource for developing individual cognitive, emotional and social capacities not well-guided.

Sexual behavior becomes the standard to look more mature and identify one from others. For adolescents in the current study, sexual behaviors of petting were deemed as appropriate. Not only do they want to act like and emulate their colleagues, but they want to show themselves by seeking to exceed their colleagues elsewhere. Given their biological changes that dictate their ague for sexual behavior, Adolescents thoroughly regulate their sexual behaviors because they feel constrained to hide them from their friends and family members. Alternatively, they opt for unidentified sexual behaviors such as necking and petting to maintain social harmony in the environment where they function.

The Social Cogitative Theory of Self-Regulation claims that adolescents govern their actions based on specific learned competencies or principles of religious principles and perceptions of what they know, their talents, knowledge of moral rules and regulations, and their cognitive abilities to create behaviors (Iskandar et al., 2013; Ridge \& Feeney, 1998). Since the social cognitive theory of selfregulation was the driving force of the present study, it is asserted that adolescents aged 19-21 at University "X" in Uganda have not yet acquired 
Muwaga, Nashori \& Sholeh | The Impact of Social Environment on The Sexual Self-Regulation of University Students in Uganda

competencies of religious values. Whereas religiosity plays an important role in adolescent sexual activities, greater individual religiosity has been correlated with a lower risk of engaging in any sexual behavior (Quinn \& Lewin, 2019), the skills and awareness concerning sexual behaviors of necking and petting, despite their strong evaluative and behavior beliefs of attitudes toward sexual behaviors, they engage in such behaviors because they lack sexual self-regulative competencies and lack clear social standards from their immediate environment of what constitute sexual behaviors, this research has immensely demonstrated that adolescents studied cannot monitor their behaviors and conditions under which behaviors of necking and petting occurs.

Adolescents evaluate such behaviors as expected and are within the limits and standards of their social environments. This situation is indicated in their strong sexual self-regulative capacities toward sexual intercourse. There are social and personal consequences attributed to such behaviors. Hines, Brook, and Conway (2004) have identified social cognitive self-regulation in individuals when they do actions that give them pleasure and self-esteem; they withdraw from activities in which they breach their moral values because such conduct brings selfcondemnation, and self-restraint holds conduct in line with internal standards.

It must be evoked that adolescents aged 19-21 in the present study freely engage in sexual behaviors of necking and petting but not intercourse and could be attributed to the fact that adolescents attitudes towards sexual behaviors were predictive of what behaviors they evaluated as fitting based on their cognitive levels of development, taking the social cognitive theory of self-regulation (Kendler, Thornton, Gilman, \& Kessler, 2000). Individuals never participate in harmful acts until they have justified the morality of their conduct on their own. In this situation, adolescents' sexual behaviors of necking and petting are morally justified and socially accepted because they serve the social worth and personal purpose of continuing with the educational pursuit and being free from disease and pregnancies in case of intercourse.

In Islam, young people who are deeply committed to their values should be less likely to compromise their religious ideals (Adamczyk \& Hayes, 2012). However, adolescents aged 19-21 at University " $X$ " in Uganda have not internalized Islamic religious values of sexual behaviors, revealing their relaxed approval of petting and necking as the behaviors that give them satisfaction. Taking this analysis further, adolescents fear social consequences from their social environment of family, university, and peers in case of intercourse than those assigned by religious teachings. One reason could be that social consequences as physical yet religious consequences are ideological of which adolescents aged 19-21 have not attained the formal operational stage of reasoning from an abstract, but their reasoning is based on concrete proof.

Adolescents aged 19-21 at "X" University also mastered evolving sexual feelings and developing a sense of sexual identity, yet this calls for life skills like sexual self-regulation. As adolescents struggle to develop their identity in the social 
Muwaga, Nashori \& Sholeh | The Impact of Social Environment on The Sexual Self-Regulation of University Students in Uganda

environment, adolescents studied lacked sexual self-regulative capacities of selfmonitoring and self-evaluation. Results from the peer environment indicate that adolescents' communication and approval of peers having sex was predicative of sexual behaviors of necking and petting because their social environment does not place constraints on such behaviors. The social environment is revealed not to influence necking and petting sexual behaviors. It may be due in part to the fact that the social environment becomes more permissive to such behaviors.

Sexual self-regulation of adolescents also revealed that their self-evaluation was negative, meaning that these individuals evaluate their sexual behaviors of necking and petting as being not as a result of their own making but factors beyond their reach, adolescents that hold defensive ideas and doubt their ability to the regulator of their behaviors lack adaptive capacities and are not committed to change because of their strong self-efficacy towards sexual behaviors of necking and petting but not intercourse.

The form of sexual behaviors that an adolescent engages in depends on a diversity of factors. Its regarded inappropriate in Ugandan culture to engage in intimate sexual behaviors and prohibit premarital childbearing before marriage (Ariho \& Kabagenyi, 2020). Even if some adolescents feel sexual urges, adolescents may limit their urges by sexual self-regulation, while, once they have come to trust each other and develop some degree of intimacy, they may engage in more sexual communication without interfering with their social environments. If this is right, the sexual self-regulatory ability will have a larger effect on an individual's sexual behavior, either by controlling his or her actions or by giving in to his or her impulses to a considerable degree, as has been seen in the present research, that all dynamics of the social environment are not crucial to adolescent sexual self-regulation.

The family environment was analyzed by Bandura and Thornberry, who concluded that bonding and contact with parents (e.g., interpersonal interaction between the parent and the child; communication patterns; parental abilities, such as supervision and discipline; parent-child conflict; and the like) had a more significant effect on the lives of adolescents, the family is the most salient arena for social interaction and involvement, with parents play a vital role in regulating the actions of adolescents, but the power of the family is diminished in the middle of puberty as the focus of youth interaction increasingly transitions from home to higher education and peer networks. The movement towards declining attachment results to parents persists as teens reach puberty, where new ways of engagement to traditional behaviors (e.g., employment) and one's family largely overtake attachment to parents and become a significant source bond to the traditional community for young adults.

Furthermore, for a family environment, religious values were significant towards adolescents' sexual self-regulation. Although adolescents religious values towards sexual behaviors were insignificant, it is essential to strengthening their sexual self-regulative capacities, linking this to the onset of adolescence 
Muwaga, Nashori \& Sholeh | The Impact of Social Environment on The Sexual Self-Regulation of University Students in Uganda

significantly increase sexual arousal and, with the example set by youth global culture companions, they are likely to begin petting as this activity provides the first ejaculation in adolescence. Since the social environment influences sexual behaviors through attitude change, sexual behaviors have consequences for unwanted pregnancies and diseases. It leaves adolescents desperate, thereby looking for other safe possible alternatives to sexual behaviors. The activity of petting among adolescents is widespread because it results in orgasm among adolescents, and the absence of social environment or religious objection to petting among teenagers whose biological changes call for sexual experimentation. By petting, adolescents preserve their virginity even though they may achieve orgasm; this means they still value virginity much as the previous generations valued it. This result is consistent with the previous study, which showed that $37 \%$ of males and $49 \%$ of females did not have sexual intercourse (Agardh, Emmelin, Muriisa, \& Östergren, 2010).

Sexual self-regulation of adolescents studied was found to be conflicting in all social environments that are family, university and peers, and it emerged that self-monitoring from an integrated composite of the social environment was $56 \%$ while self-efficacy was slightly higher with $82 \%$ and self-evaluation $-54 \%$. These results reveal that adolescents aged 19-21 at University " $X "$ their sexual self-regulative capacities are in negatives that are $-56 \%$, meaning that these people cannot structure their environment through searching for information and rejecting deviant peers. A simple glance at self-efficacy revealed that adolescents aged 19-21 at University "X" in Uganda, their self-efficacy emerged to be $82 \%$, meaning that these adolescents are willing to monitor and structure their environment for appropriate sexual behaviors. However, these adolescents lack the adaptive capacities of self-evaluation, that is $-54 \%$. This result means that despite their self-efficacy to monitor their behaviors and environment, they are self-handicapped because they fear losing their friends who engage in sexual behaviors and seeking sexual information from their social environment that would render them promiscuous.

This study still has several limitations involving limited subjects, so it was not easy to be generalized to the existing populations. It is crucial to pick variables carefully to completely cover the domain of sexual self-regulation since experiments have been appropriate with a few components and a small sample design and are thus flawed due to a lack of sampling of both variables and various subjects relevant to the topic of sexual self-regulation.

\section{CONCLUSION}

The findings of the study demonstrated how social environments significantly influenced adolescent's sexual self-regulation. The social environment significantly influences adolescent sexual self-regulation through modification of attitudes. The absence of a significant relationship between social environment and sexual self-regulation is attributed to Ugandan culture, where discussion or 
Muwaga, Nashori \& Sholeh | The Impact of Social Environment on The Sexual Self-Regulation of University Students in Uganda

explicit information about sex is regarded as taboo. However, results demonstrated that social environments indirectly influence adolescents' sexual self-regulation through religious values and attitudes for a family environment.

Longitudinal research aiming at developing sexual self-regulation skills among adolescents is strongly recommended. The environment in which adolescents are trained to encourage sexual self-regulation during their education needs to be investigated.

\section{REFERENCES}

Adamczyk, A., \& Hayes, B. E. (2012). Religion and sexual behaviors: Understanding the influence of Islamic cultures and religious affiliation for explaining sex outside of marriage. American Sociological Review, 77(5), 723746. https:/ / doi.org/10.1177/0003122412458672

Agardh, A., Emmelin, M., Muriisa, R., \& Östergren, P.-O. (2010). Social capital and sexual behavior among Ugandan university students. Global Health Action, 3(1), 5432. https:/ / doi.org/10.3402/gha.v3i0.5432

Ariho, P., \& Kabagenyi, A. (2020). Age at first marriage, age at first sex, family size preferences, contraception and change in fertility among women in Uganda: analysis of the 2006-2016 period. BMC Women's Health, 20(1), 8. https:/ / doi.org/10.1186/s12905-020-0881-4

Barnett, E., \& Casper, M. (2001). A definition of "social environment." American Journal of Public Health, 91(3), 465a - 465. https://doi.org/10.2105/ AJPH.91.3.465a

Blanchard, R., Zucker, K. J., Siegelman, M., Dickey, R., \& Klassen, P. (1998). The relation of birth order to sexual orientation in men and women. Journal of Biosocial Science, 30(4), 511-519. https:// doi.org/10.1017/S0021932098005112

Fried, R. A. (2015). No Irish Need Deny: Evidence for the Historicity of NINA Restrictions in Advertisements and Signs. Journal of Social History, 49(4), 829854. https:/ / doi.org/10.1093/jsh/shv066

Frost, D. M., \& Meyer, I. H. (2009). Internalized homophobia and relationship quality among lesbians, gay men, and bisexuals. Journal of Counseling Psychology, 56(1), 97-109. https://doi.org/10.1037/a0012844

Hayes, D., Blake, J. J., Darensbourg, A., \& Castillo, L. G. (2015). Examining the academic achievement of Latino Adolescents. The Journal of Early Adolescence, 35(2), 141-161. https:/ / doi.org/10.1177/0272431614530806 
Muwaga, Nashori \& Sholeh | The Impact of Social Environment on The Sexual Self-Regulation of University Students in Uganda

Hines, M., Brook, C., \& Conway, G. S. (2004). Androgen and psychosexual development: Core gender identity, sexual orientation, and recalled childhood gender role behavior in women and men with congenital adrenal hyperplasia (CAH). Journal of Sex Research, 41(1), 75-81. https:// doi.org/10.1080/00224490409552215

Iskandar, Z., Setiono, K., Agustiani, H., \& Musa, M. (2013). The influence of value systems and sexual self-regulation towards adolescent's sexuality. Indian Journal of Positive Psychology, 4(2), 315-320. https:// doi.org/10.15614/ijpp/2013/v4i2/49914

Kendler, K. S., Thornton, L. M., Gilman, S. E., \& Kessler, R. C. (2000). Sexual orientation in a U.S. national sample of twin and nontwin sibling pairs. American Journal of Psychiatry. https://doi.org/10.1176/appi.ajp.157.11.1843

Lauren, W., \& Sharlene, S. (2012). adolescence. In J. Hardman (Ed.), Child and adolescent development: A South African sociocultural perspective (pp. 203-244). Oxford University Press.

Leung, H., Shek, D., Leung, E., \& Shek, E. (2019). Development of contextuallyrelevant sexuality education: Lessons from a comprehensive review of adolescent sexuality Education Across Cultures. International Journal of Environmental Research and Public Health, 16(4), 621. https://doi.org/10.3390/ijerph16040621

Mikami, A. Y., Ruzek, E. A., Hafen, C. A., Gregory, A., \& Allen, J. P. (2017). Perceptions of relatedness with classroom peers promote adolescents' behavioral engagement and achievement in secondary school. Journal of Youth and Adolescence, 46(11), 2341-2354. https://doi.org/10.1007/s10964017-0724-2

Mujiyati, M., \& Adiputra, S. (2018). Influence of peer groups to the self-esteem of Lampung and Javanese Students. International Journal of Psychology and Educational Studies, 5(1), 15-22. https://doi.org/10.17220/ijpes.2018.01.003

Noviva, H., \& Wahyono, T. Y. M. (2020). Factors associated with risky sexual behavior in adolescent boys in Indonesia. Proceedings of the 2nd Sriwijaya International Conference of Public Health (SICPH 2019). https:// doi.org/10.2991/ahsr.k.200612.040

Quinn, D., \& Lewin, A. (2019). Family religiosity, parental monitoring, and emerging adults' sexual behavior. Religions, 10(2), 114. https:// doi.org/10.3390/rel10020114

Ragelienè, T. (2016). Links of adolescents identity development and relationship 
Muwaga, Nashori \& Sholeh | The Impact of Social Environment on The Sexual Self-Regulation of University Students in Uganda

with peers: A systematic literature review. In Journal of the Canadian Academy of Child and Adolescent Psychiatry.

Ridge, S. R., \& Feeney, J. A. (1998). Relationship History and Relationship Attitudes in Gay Males and Lesbians: Attachment Style and Gender Differences. Australian \& New Zealand Journal of Psychiatry, 32(6), 848-859. https:// doi.org/10.3109/00048679809073875

Rosario, M., Schrimshaw, E. W., Hunter, J., \& Braun, L. (2006). Sexual identity development among lesbian, gay, and bisexual youths: Consistency and change over time. Journal of Sex Research, 43(1), 46-58. https:// doi.org/10.1080/00224490609552298 